Edited by Kiriakos Xenitidis and
Colin Campbell
Contents
- Deinstitutionalisation, imprisonment
and homelessness
- Trial of an intervention to reduce suicidal
ideation and behaviour
- Evolutionary theories in disordered eating
psychopathology

\section{Deinstitutionalisation, imprisonment and homelessness}

In May 2016, Winkler et al published a systematic review on cohort studies following up patients after discharge from longterm psychiatric hospital care. ${ }^{1}$ The study did not show relevant numbers for imprisonment or homelessness after discharge. The authors concluded that the study contradicted ecological studies reporting a relationship between prison population rates and psychiatric bed numbers. They propose ecological fallacies as a possible explanation. In the related editorial, the ecological studies are referred to as arguing against deinstitutionalisation. ${ }^{2}$

As an author of one of those ecological studies, I would like to comment. Rather than arguing against deinstitutionalisation of mentally ill people, the studies express concern that deinstitutionalisation does not occur in societies with massively increasing prison populations ${ }^{3}$ and very high rates of severe mental illness among prisoners. ${ }^{4}$ Although the relationship between psychiatric bed numbers and prison population rates in South America was rather strong, findings from Europe were less robust. ${ }^{5}$ In quantitative terms, prisons have become the most important facilities institutionalising mentally ill people in the Americas. Mentally ill people in prisons cause much more concern with respect to human rights than those in psychiatric hospitals. A way forward could be to improve care for people in prison, as well as improving community care for mentally ill people at risk of criminal justice involvement to prevent imprisonment. There is a broad consensus that short-term hospital admission is more efficient than longer stays, and that psychiatric hospital admission should be linked with community services in care systems. In the ecological studies, all types of psychiatric hospital beds were acknowledged. $^{3}$ The majority of beds nowadays are used to provide short-term care, including in low- and middle-income settings. Long-term hospital admission is no longer a common type of service provision in general psychiatry in the countries in which the studies that Winkler et al included in the review were conducted. ${ }^{1}$ Therefore, the study seems rather of historical value.

It is not surprising that elderly people, after decades spent in hospital, have low criminogenic energy. Young people with severe mental illness and comorbid substance use disorders are of much more concern. For understanding the interdependence of penal justice systems and psychiatric in-patient care systems, recently published large linkage studies of registries are more relevant. These show very high rates of psychiatric hospital admission prior to imprisonment and in the year after release from imprisonment. They also show markedly elevated risks for people with mental disorders to commit violent crimes and to be victims of violence compared with the general population. What contribution shortterm hospital care can make to postponing or preventing criminal justice involvement and protecting people with mental disorders is still unresolved. However, to reject findings from ecological studies, as in Winkler et al's review, may be a fallacy of categories.

1 Winkler $P$, Barrett $B$, McCrone $P$, Csémy L, Janoušková $M$, Höschl C. Deinstitutionalised patients, homelessness and imprisonment: systematic review. Br J Psychiatry 2016; 208: 421-8.

2 Salisbury $\pi$, Thornicroft G. Deinstitutionalisation does not increase imprisonment or homelessness. Br J Psychiatry 2016; 208: 412-3.

3 Mundt AP, Chow WS, Arduino M, Barrionuevo $H$, Fritsch $\mathrm{R}$, Girala $\mathrm{N}$, et al. Psychiatric hospital beds and prison populations in South America since 1990: does the Penrose hypothesis apply? JAMA Psychiatry 2015; 72: 112-8.

4 Mundt AP, Kastner S, Larrain S, Fritsch R, Priebe S. Prevalence of mental disorders at admission to the penal justice system in emerging countries: a study from Chile. Epidemiol Psychiatr Sci 2015; doi: 10.1017/ S2045796015000554.

5 Chow wS, Priebe S. How has the extent of institutional mental healthcare changed in Western Europe? Analysis of data since 1990. BMJ Open 2016; 6: e010188

Adrian P. Mundt, Psychiatrist, Medical Faculties Universidad Diego Portales, Universidad San Sebastián and Universidad de Chile. Email: adrian.mundt@mail.udp.cl doi: 10.1192/bjp.209.4.349

As the authors of a previous review of deinstitutionalisation and homelessness, ${ }^{1}$ we were interested to see our 1992 findings confirmed by Winkler et al's recent paper $^{2}$ and the accompanying editorial. ${ }^{3}$ We particularly agree with the notion that apparent relationships between deinstitutionalisation and homelessness can often be mediated by substantial confounding factors. In London in the 1980s, it was the unheralded and unpublicised closure of most of the city's homeless hostel beds that seemed the most likely culprit.

Although this issue may well still be pertinent in other healthcare and social systems, it was of decreasing relevance in the UK even when we published our paper in 1992. The process of deinstitutionalisation was, by then, irreversible and substantially accomplished. This leads us to our concern that these papers might support an unhelpful sense of complacency.

Taylor Salisbury \& Thornicroft's statement that 'instances of homelessness ... among those discharged are rare ${ }^{3}$ is clearly correct in referring to the institutional closures and hospital discharges that are now several decades in the past. However, it is at odds with the situation of hospital discharge as it stands today, at least in London. I work in a psychiatric outreach team for homeless people in South London, where homelessness following hospital discharge is common among referrals to our service. We looked at 3 months of our referral data last year and found that $60 \%$ of our homeless referrals (mainly with a diagnosis of psychosis) had had previous contact with our local mental health service. They had had, on average:

- contacts with 4 separate trust services

- 35 contacts (face-to-face/phone triage), 2 of these would have been emergency contacts, seen in an accident and emergency department, or in a section 136 suite

- 65 days as an in-patient in the local trust service.

These people had sometimes been discharged to the street, or referred to local community services, but without effective plans to prevent them becoming homeless again. We note that observations we made in 1992 still stand - the excessive bed occupancy of in-patient services is driving an emphasis on short episodes of in-patient treatment. 